\title{
Single Server Queuing Model to Determine the Patient Flow Process in a Township Hospital
}

\author{
Vijaya Mani, Vani Haridasan
}

\begin{abstract}
This research aims to analyse the use of queuing theory in the two branches of a local township health care centre located in a small township in Tamil Nadu, India. A scenario from the out-patient departments of the aforesaid centre shows the relationship between the different variables operating the system. The focus of this research is also to provide some insights for improving the efficiency of the medical centre through the queuing model. The research concludes that the Queuing system at branch $A$ of the health centre is $93 \%$ efficient and at Branch $B$ it is $73 \%$ efficient.
\end{abstract}

Key Words: Queuing, Waiting time, Poisson, FIFO, Traffic Congestion

\section{INTRODUCTION}

Queuing theory the study of waiting lines (Adeleke, R. A., Ogunwale, O. D., \& Halid, O. Y. (2009) .It is a system which involves individuals customers requiring some service which has some restriction. Bailey did a significant research on the usage of queing theory in a healthcare setting (Bailey, 1952). He developed an out-patient scheduling system that gave acceptable results for patients (in terms of waiting time) and doctors (in terms of utilisation). Adeleke R. A et al (2009) applied queuing theory to determine the waiting time of out-patients in a hospital.

To make health care system more efficient and economical, queuing theory can be applied. From 1952, usage of queuing theory is being researched in the medical and health care systems. McClain had done research on optimizing the bed utilization and staff assignments in the hospitals. Praetor has emphasized the importance of the use of queuing theory in health care. Agnihotri, S.R and Taylor, P.F. Khan, M.R. and Callahan, B.B also has contributed to the research in queuing applications.

\section{RESULTS AND DISCUSSION}

The research was carried out at a local secondary health care centre (Branch A) and Branch (B) located in a small town in Tamil Nadu (India). The study is based on the data collected through observation and face to face interaction with the patients. The aim of the study is to decrease the waiting lines of the patients in both the branches and in turn increase the effectiveness. At the registration counter two persons are deputed for providing registration services and they are given their weekly schedule at the beginning of each week.

\section{Branch A}

\footnotetext{
Revised Manuscript Received on September 14, 2019.
}

Vijaya Mani, SSN School of Management, Kalavakkam, Tamil Nadu, India.

Vani Haridasan, SSN School of Management, Kalavakkam, Tamil Nadu, India.
The patients usually arrive from 8:30am. A total of 1092 patients arrived for service during this four weeks of observation (March 2019) for 22 working days. Considering the unit of time for the model as one day of 8 hours working, the total number of patient's arrival and the mean for the arrivals at different intervals were calculated to be $\lambda=$ 50 per day. The patients have to follow a protocol. Once they finish the registration, they are given a token no, with which they have to meet the doctor. Some patients are prescribed the medicines and asked to come later for a review, whereas others are just told to follow the doctor's advice and take rest. The data for the research is collected by observing the patients and through personal interaction by the researcher.

The waiting time of the patients is calculated by subtracting the arrival time of patients from the time of the start of service every day. The patients usually arrive before the time the service at the registration counter begins every working day. The health center had only one type of service; OPD Consultancy. The doctor offers his service as and when a patient arrives starting from 8:30am every day. At total of 1092 patients were served during the four weeks of observation. The total number of patients served by the doctor and the mean of service rendered at different time intervals were calculated. The mean service rate was calculated to be $\mu=54$ patients per day.

Calculations based on Single Queuing Model:

Total Working Days: 22

Total Patients: 1092

Total waiting time $=1680$ minutes

Total service time $=1220$ minutes

(i) Unit of time $=1$ day is equal to 8 hours

(ii) The arrival rate $\lambda=50$ patients per day

(iii) The service rate, $\mu=54$ patients per day

(iv) The average time spent by patients in the queue $\mathrm{W}_{\mathrm{q}}=\lambda / \mu(\mu-\lambda)=13.89$ minutes $\sim 14$ minutes

(v) The average time spent by the patients in the system

$$
\mathrm{W}_{\mathrm{s}}=1 /(\mu-\lambda)=15 \text { minutes }
$$

(vi) Average number of patients in the system

$$
\mathrm{L}_{\mathrm{s}}=\rho /(1-\rho)=\lambda(\mu-\lambda)=12.5 \sim 13 \text { patients }
$$

(vii) Average queue length

$$
\mathrm{L}_{\mathrm{q}}=\lambda^{2} / \mu(\mu-\lambda) \quad=11.57 \quad \sim 12 \text { patients }
$$

(viii) Utilization factor

$$
=\rho=\lambda / \mu=0.93 \text { or } 93 \%
$$




\section{Branch B}

The patients usually arrive from 8:30am. A total of 798 patients arrived for service during this four weeks of observation (March 2019). The total number of patient's arrival and the mean for the arrivals at different intervals were calculated to be $\lambda=38$ patients per day. The patients have to follow a protocol. Once they finish the registration, they are given a token no, with which they have to meet the doctor. Some patients are prescribed the medicines and asked to come later for a review, whereas others are just told to follow the doctor's advice and take rest. The data for the research is collected by observing the patients and through personal interaction by the researcher.

The waiting time of the patients is calculated by subtracting the arrival time of patients from the time of the start of service every day. The patients usually arrive before the time the service at the registration counter begins every working day. The health center had only one type of service; OPD Consultancy. The doctor offers his service as and when a patient arrives starting from 8:30am every day. The arrival time as well as the time service began and ended for 798 patients in the Branch B of the local health care centre. When service is available, service is made by the doctor immediately a patient arrives therefore, service are rendered to the patients at the same time of arrival, starting from 8:30am every day. At total of 798 patients were served during the four weeks of observation. The total number of patients served by the doctor and the mean of service rendered at different time intervals was calculated. The mean service rate was calculated to be $\mu=45$ patients per day.

Total waiting time of 798 patents for 22 days $=1680$ minutes

Total service time of 798 patients for 22 days $=1220$ minutes

(i) Unit of time $=8$ hours

(ii) The arrival rate $\lambda=38$ patients per day

(iii) The service rate, $\mu=45$ patients per day

(iv) The average time incurred by patients in the queue $\mathrm{W}_{\mathrm{q}}=\lambda \mu(\mu-\lambda)=7.24$ minutes $\quad \sim 7$ minutes

(v) The average time incurred by patients in the system

$$
\mathrm{W}_{\mathrm{s}}=1 /(\mu-\lambda)=8.57 \text { minutes } \sim 9 \text { minutes }
$$

(vi) Average number of patients in the system

$$
\mathrm{L}_{\mathrm{s}}=\rho /(1-\rho)=\lambda(\mu-\lambda)=5.43 \sim \text { 6patients }
$$

(vii) Average length of the queue

$$
\mathrm{L}_{\mathrm{q}}=\lambda^{2} / \mu(\mu-\lambda) \quad=4.58 \quad \sim 5 \text { patients }
$$

(viii) Utilization factor

$$
=\rho=\lambda / \mu=.84=84 \%
$$

\section{CONCLUSION}

The research has focussed on a single channel queuing system to represent the two branches of a local health centre where the doctor is considered as a single server. The research case is based on the data collected through observation and face to face interaction with the patients by the researcher during the four weeks (March 2019) for 22 working days from 1092 patients at Branch A and 798 Patients at Branch B. The traffic intensity $=\rho=\lambda \mu=0.93$ at Branch A works out to be $93 \%$. The results show that the traffic intensity at the Branch A of the health centre is quite good currently. The traffic intensity $=\rho=\lambda / \mu=.84$ at Branch B states the probability of patients queuing on arrival. It works out to be $84 \%$.. It has also been seen from the analysis that at Branch $\mathrm{B}$, the average time spent in the centre (in queue and in receiving treatment) is greater than the average time spent in the queue before providing treatment. Thus, it can be concluded that there will always be a queue of patients in the Branch $\mathrm{B}$ of the centre. The results also shows that there is a very less possibility of providing services to newly arriving patients. This Queuing system can be improved either by deputing one more server that is a doctor or an attendant for the check up of the patients or the single server that is the doctor's clinic time be increased so that he /she may be able to reduce the queue and make the hospital services better for the patients.

\section{REFERENCES}

1. Adeleke, R. A., Ogunwale, O. D., \& Halid, O. Y. (2009), Application of Queuing Theory to Waiting Time of OutPatients in Hospitals", Pacific Journal of Science and Technology , 10(2):270-274.

2. A.K. Erlang, The Theory of Probabilities and Telephone Conversations, Nyt Tidsskrift for Mate matik, 20(B), 1909, 33-39.

3. Blake, F. I. (1978), An Introduction to Probability Models. USA: John Wiley and Sons Inc.

4. Bailey N. T. J. (1952), A study of queues and appointment systems in hospital out- patient departments, with special reference to waiting-times, Journal of the Royal Statistical Society. Series B (Methodological), 14(2):185-199,

5. Churchman, G. W., Ackoff, R. C., \& Arnoff., F. C. (1957). Introduction to Operation Research. New York, NY: John Willey and Sons:.

6. S.R. Agnihotri and P.F. Taylor, Staffing a centralized appointment scheduling department in Lourdes Hospital, Interfaces-Journal Online, 21, 1991, 1-11. 